\title{
Microbial Interactions with Nanostructures and their Importance for the Development of Electrospun Nanofibrous Materials used in Regenerative Medicine and Filtration
}

Chris J Mortimer, Luke Burke and Chris J Wright*

Biomaterials, Biofouling and Biofilms Engineering Laboratory (B3EL), Systems and Process Engineering Centre, College of Engineering, Swansea University, Fabian Way, Swansea, UK

\begin{abstract}
The growing use of electrospinning to fabricate nanofibrous structures for use in wound dressings, tissue engineering and filtration processes has increased the need for an understanding of the interactions between bacteria and nanostructures. The adhesion characteristics and colonisation of bacteria on these materials is still not completely understood but is essential to aid their future development. This review presents the state of current research on microbial attachment at materials with micro- and nano- structures and how this research has been developed and adapted to study the interactions of bacteria with nanofibres. The few studies to date are discussed with the view to identifying the future studies required to increase understanding and allow the technology and application of electrospun nanofibres to move forward.
\end{abstract}

Keywords: Nanofibres; Electrospinning; Bacteria; Biofilm; Adhesion; Nanostructures; Antimicrobial; Antibiofilm

\section{Introduction}

Electrospinning is rapidly becoming a common technique for the fabrication of non-woven fibrous structures for applications in tissue engineering, wound healing and filtration [1-7]. This is due to the ability to electrospin fibres on both the nano- and micro-scale from synthetic and naturally occurring biocompatible polymers (Figure 1). An electrospun fibre mesh offers many desirable properties such as a high surface area to volume ratio, potential for drug release including antimicrobials, controllable fibre diameters, high porosity and permeability. In addition, modern electrospinning techniques have emerged that ensure the fabrication technique is scalable and so economically viable for high volume manufacture.

Electrospinning is an area that has been subject to a vast amount of research due to the technique's facile nature and ability to rapidly develop polymer constructs of fibres with a diameter on the nanoscale. Recently there has been renewed interest in the technology due to increased demand from regenerative medicine. This is accompanied by improved fabrication capabilities and process optimisation through surface characterisation permitted by nanotechnology. Regenerative medicine is looking to exploit the advantages of electrospinning in the creation of 3-D scaffolds and dressing materials with improved delivery of biologically active materials including antibiotics to maintain environments with a low bacterial loading. Electrospinning as a technique was first documented by L. Rayleigh in 1878 [8], with the first patents filed by J.F. Cooley [9]. Electrospinning uses a high voltage power supply to create a large potential difference between a grounded "collector" structure and a polymer solution or melt being delivered at a constant rate through an aperture, such as a blunt end needle (Figure 1a). As the voltage is increased the like charges within the polymer fluid directly oppose surface tension, resulting in the normally spherical droplet at the aperture distending into a conical shape. This cone is referred to as the "Taylor" cone, after Sir Geoffrey Taylor who first mathematically modelled the phenomenon [10-12]. At a critical voltage the electrostatic attractive force between the solution and the collector causes a jet of polymer solution to be expelled from the cone tip towards the grounded collector surface. This jet then undergoes a whipping instability and dries in flight, depositing the nanofibres on the collector [2]. Modifications to the electrospinning process such as multi needle electrospinning [13] and free surface electrospinning $[14,15]$ have offered vastly increased production rates and opened up many further applications due to their scalability (Figure 1b).

Electrospun fibres have been thoroughly researched for tissue engineering applications. A tissue engineering scaffold needs to have a sufficient pore size and porosity to allow the proliferation of cells and a sufficient surface area to volume ratio to promote cell adhesion, growth migration and differentiation [16]. For this reason it is very important to understand the relationship between cells and nanofibrous structures. Although there has been a vast amount of research on the interactions between nanofibres and eukaryotic cells $[17,18]$ there has been very little research focussed on the interaction of micro-organisms with nanofibres [19]. An improved understanding of the fundamental processes involved in such interactions is essential to aid the further development of nanofibre mats for application in environments such as wound dressings, filtration and tissue engineering that can all be compromised by microbial colonisation. This short review will examine the current state of research based on bacterial-fibre interactions, identify shortfalls in our understanding and address why such knowledge is necessary to move the technology forward.

*Corresponding author: Chris $\mathrm{J}$ Wright, Biomaterials, Biofouling and Biofilms Engineering Laboratory (B3EL), Systems and Process Engineering Centre, College of Engineering, Swansea University, Fabian Way, Swansea, UK, Tel: +44 (0) 1792 295200; E-mail: c.wright@swansea.ac.uk

Received March 18, 2016; Accepted April 22, 2016; Published April 29, 2016

Citation: Mortimer CJ, Burke L, Wright CJ (2016) Microbial Interactions with Nanostructures and their Importance for the Development of Electrospun Nanofibrous Materials used in Regenerative Medicine and Filtration. J Microb Biochem Technol 8: 195-201. doi: 10.4172/1948-5948.1000285

Copyright: (c) 2016 Mortimer CJ, et al. This is an open-access article distributed under the terms of the Creative Commons Attribution License, which permits unrestricted use, distribution, and reproduction in any medium, provided the original author and source are credited. 
(A)

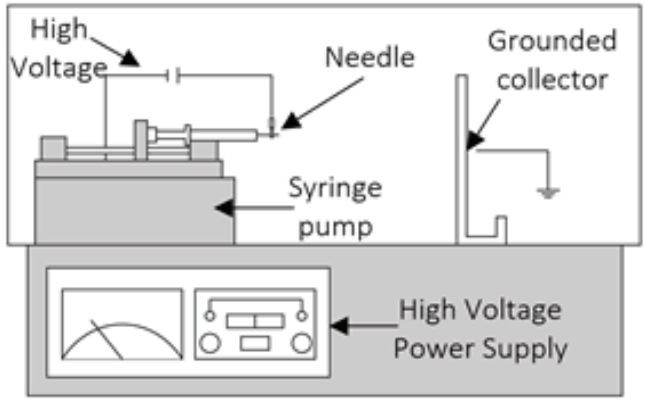

(B)

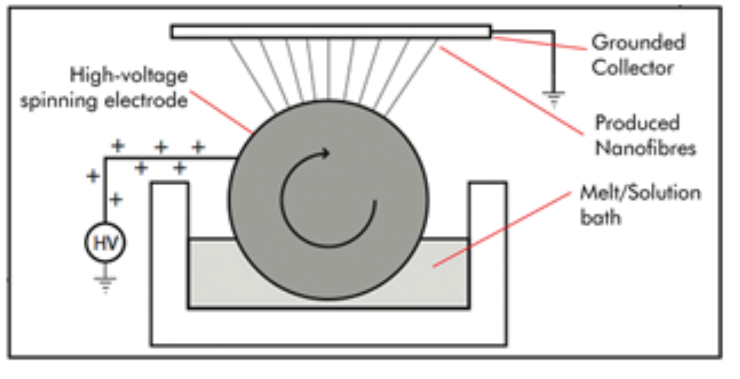

Figure 1: A schematic diagram showing a basic needle-based electrospinning set-up (A) and free-surface electrospinning set-up (B). In a needle electrospinning set-up a high voltage power supply is used to extrude fibres from a polymer solution/melt being drawn through a blunt end needle using a syringe pump. Fibres are collected on a grounded plate. In a free-surface electrospinning set-up a polymer solution/melt is held in a bath and a spinning electrode connected to a high voltage power supply is utilized to form multiple jets. Nanofibers are electrospun upwards and collected on a grounded collector plate.

\section{Application and Importance of Electrospun Nanofibrous Materials}

The fabrication of nanofibres has been exploited in the manufacture of many materials for a wide range of industrial applications. However, it is in separation processes and the clinical sector that the interaction of microbial populations with nanofibres has arguably the greatest impact. In regenerative medicine a number of polymers have been used and include poly (lactic-co-glycolic) acid (PLGA) [20], Polycaprolacetone (PCL) [21], polyurethane (PU) [22], Polyvinyl alcohol (PVA) [23] and collagen [24] . Anselme discussed the importance of understanding the interaction between bacteria and nanostructures and identified this to be of key importance when developing tissue engineering scaffolds as cells have to compete with bacteria in many environments and structures must both promote cell adhesion while inhibiting bacterial cell adhesion [25]. This is a research intensive wish for many medical devices that are required to integrate into tissues by encouraging interaction with the human cells yet discouraging interaction with bacteria, which gain significant advantage at the surface of implants forming biofilms that evade treatment. Nanofibrous materials have gained favour with tissue engineers as they have a structure that is analogous to the extracellular matrix found in many tissues $[5,26,27]$. A scaffold that mimics the cellular niche and encourages cellular integration is optimum for tissue engineering. Nanofibres that have been functionalised to promote human cell adhesion are key materials and as identified by Anselme there is a caveat that the material should not encourage bacterial colonisation [25]. To attain this, nanofibres can also be functionalised with antimicrobials [28]. However, control may be achieved as a result of the morphology of nanofibrous materials. Eukaryotic cells are more deformable allowing them to have more interaction with nanostructures, as deformation on attachment and subsequent cell growth increases the surface area contact; they will contact a number of fibres in the material structure. In contrast bacteria are smaller and far more rigid structures, there will be far less deformation on contact and the interaction will be at a smaller number of fibres (This is further discussed in section 1.5).

In another aspect of regenerative medicine, wound healing, electrospinning is now an established technique for the production of wound dressings for the treatment of chronic wounds [6,29-35]. Many modern wound dressings are no longer only a barrier to protect a wound from infection but they are also expected to enhance the healing of the wound [36]. To achieve this research has looked to electrospun materials to enable this multifunctionality. A wound dressing should be selected based on the type of wound and an ideal wound dressing should maintain a moist environment, remove exudates, prevent microbial infection and allow gaseous exchange [6]. Unlike acute wounds, chronic wounds can often take over 12 weeks to heal and it is not uncommon for these wounds to reoccur [37]. As such chronic wounds are a major burden on health services across the world with antimicrobial resistance resulting in longer treatment terms and increased infection rates. Immune response due to bacteria being present can delay healing due to tissue inflammation [36]. Infection can lead to the formation of a biofilm making treatment more difficult and further prolong healing. Normal dressings generally are not suitable for the treatment of chronic wounds, which has encouraged the development of bioactive dressings. These dressings provide a suitable environment to aid in the healing of the wound by altering the chemical environment [36]. For these applications fibrous materials are often desirable. Nanofibres as part of a dressing act as a physical barrier offering protection from bacteria migrating to the wound. This will reduce the bioburden and the likelihood of biofilm formation at the site of the wound. To this end, nanofibre wound dressings have been functionalised with antimicrobial agents including antibiotics, biocides and silver $[36,38,39]$. This not only further prevents passage of contaminating bacteria that colonise the external surface of the dressing, but in some cases the functionalised nanofibre dressing can also act as a reservoir that releases antimicrobials into the wound bed to control bacteria already present and reduce biofilm on patient tissues. To further develop the use of nanofibres for wound dressings a further understanding of the interactions between bacteria and nanofibres is required which will aid in producing wound dressings that are capable of inhibiting the adhesion of bacteria and decreasing colonisation.

Fibrous materials have been used extensively in the fabrication of synthetic membranes used in separation processes [40]. By controlling the fibre diameter different pore sizes can be obtained making them useful for many applications. Focusing on air filtration where removal of microorganisms is an important efficiency indicator, electrospun nanofibre mats can be used due to their small diameter fibres and small pore size allowing them to trap particles while allowing sufficient air flow. Filters have been electrospun from many synthetic and biopolymers such as nylon 6 for use as high efficiency air particulate (HEPA) filters and ultra-low particulate air (ULPA) filters [41], polyethylene oxide (PEO) for use in composite filters for long range applications [42], polyacrylonitrile (PAN) for nanoparticle filtration [43] and a composite of Polylactide/polyhydroxybutyrate (PLA/PHB) for aerosol particle filtration [44]. One major problem with the very 
small pore size of nanofibrous filters is their bacterial contamination leading to a short life span. Bacterial contamination can therefore become a problem with bacterial adhesion resulting in colonisation and biofilm formation. An understanding of bacterial interaction with nanofibres is therefore essential to progress the development of nanofibrous air filtration systems, to aid the design and optimisation of antimicrobial control strategies.

\section{Interaction of Bacteria with Nanostructures}

Adhesion of bacteria is one of the early stages of biofilm formation occurring after or alongside the laying down of a conditioning film depending on the environment of the surface. Biofilms are now widely regarded as the predominant mode of microbial life in both nature and disease due to their inherent resistance to antimicrobials and ability to adapt and 'protect' themselves from attack [45-48]. How the cells initially interact in surface adhesion is therefore a very important factor when developing antimicrobial and antibiofilm surfaces for application in medicine and the process industries. In recent years there has been extensive research into the adhesion mechanisms of bacteria with more recent studies focussing on the interaction between bacteria and textured surfaces; surfaces that have controllable nanoscale morphology. The use of surfaces with micro and nanosurfaces, which have the potential to control bacterial adhesion and subsequent biofilm formation is another technique in the portfolio of methods available to control microbial attachment. The mode of action of such an approach is to reduce the area of surface interaction and the number of participating bonds, thus rendering the initial bacterial attachment more vulnerable to physical methods of disruption such as hydrodynamic shear [49]. Prevention of attachment through physical means compliments or removes the need for chemical methods.

Otto reviewed the biophysical approaches used to study the dynamic process of bacterial adhesion [50]. The researcher identified two major achievements in understanding the molecular mechanisms of bacterial adhesion; Genetic screens for adhesion-deficient mutants using microtiter dish assays [51] and the combination of confocal laser scanning microscopy and digital image analysis [52]. This helped in understanding the difference between biofilms and planktonic cells. Due to the limitations of these techniques novel non-invasive methods have been investigated enabling adhesion measurements at the single cell level $[53,54]$. These methods can complement each other together with molecular biological techniques to increase our understanding of the molecular process behind adhesion and aid in developing methods to inhibit the adhesion of bacteria as an antimicrobial approach. These techniques have proven to be important to assessing the adhesion of microorganisms to nanostructures. In another important report in 2010 Anselme reviewed the interaction of cells and bacteria with surfaces structured at the nanoscale and they concluded that the way in which cells interact with a surface on the nanoscale is the same as at other scales [25]. However, patterning on the nanoscale is likely to have a significant impact with the organisation of attachment points playing an important role in bacterial cell response to topography. It is important that binding sites are available on the material surface for the bacteria to contact and achieve their initial attachment [55]

Whitehead studied the retention of bacterial cells on substrates with micro- and sub-micrometre dimensions finding that a larger number of cells accumulated in structures with larger round surface features ( 1 and $2 \mu \mathrm{m}$ diameter) when compared with smaller round surface features ( 0.2 and $0.5 \mu \mathrm{m}$ diameter) [56]. They used irregularly spaced surface pits of regular features $(0.2$ and $0.5 \mu \mathrm{m})$ and regularly spaced pits with regular features ( 1 and $2 \mu \mathrm{m}$ diameter). These substrates were subjected to retention assays. Staphylococcus aureus were retained in the highest numbers, particularly within the $0.5 \mu \mathrm{m}$ pits. Pseudomonas aeruginosa were preferentially retained within the $1 \mu \mathrm{m}$ surface features and Candida albicans blastospores were retained in $2 \mu \mathrm{m}$ pits. Both $S$. aureus and $P$. aeruginosa were retained in the greatest number by the largest $2 \mu \mathrm{m}$ surface feature. The number of retained C. albicans cells was found to be similar across all surfaces. In a similar study Edwards investigated the role of micro topographical surface features of sulphide minerals in localising and aligning bacterial adhesion [57]. They found that the depth of surface feature is less important than its cross-sectional shape. They concluded that the micro topography can significantly alter binding strength and is of particular importance when the size and shape of the topography is very similar to that of the bacterial cell. They also argued that any alteration of the surface topography by the metabolism of the bacteria is important as this could increase the likelihood of adhesion. In another key study the adhesion of $P$. fluorescens on nano/microengineered sufaces was studied by Diaz [58]. Gold substrates with different surface structures were prepared using physical vapour deposition (PVD) along with moulding and replication techniques. Substrates were immersed in an overnight culture of $P$. fluorescens. The bacteria formed well-defined clusters on the substrates of the study. On the randomly nanostructured surfaces bacterial clusters had a densely packed structure with a small number of isolated bacteria this was compared to bacterial clusters with an open structure and a larger number of isolated bacteria on the parallel trenches of another substrate showing the influence of the substrate nanostructure on bacterial colonisation (Figures 2 and 3 ). Bacteria lay across the peaks of ripples on a third substrate as the width and depth of the features were significantly smaller than the cells. Chung studied the impact of an engineered surface micro/nano topography mimicking the structure of shark skin on the formation of $S$. aureus biofilms [59]. The substrate consisted of $2 \mu \mathrm{m}$ wide rectangular ribs of lengths ranging from 4 to 16 $\mu \mathrm{m}$. The ribs were arranged on PDMSe surfaces with a fixed spacing of $2 \mu \mathrm{m}$ between features. It was found that micro colonies of bacteria formed on smooth surfaces on day 2 with only isolated cells between features on the textured surface. Biofilm formation started on smooth samples earlier, showing evidence of mature biofilm formation at day 14 with only a slight increase in cell clusters on textured surfaces with no evidence of biofilm formation. After 21 days biofilms colonised the

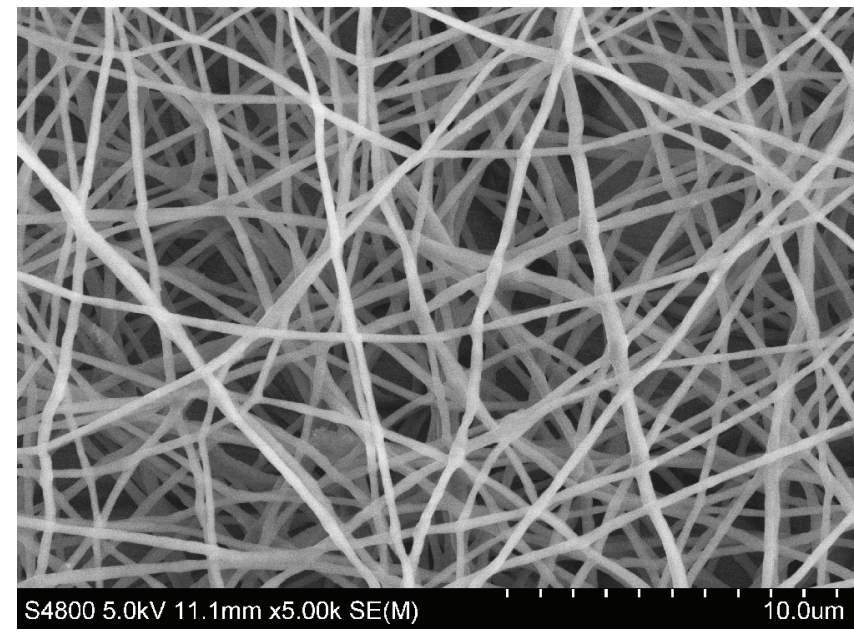

Figure 2: An SEM image showing Poly (ethylene oxide) nanofibres electrospun using a needle based electrospinning set up. 
majority of the smooth PDMS surface and the textured surfaces showed the first signs of biofilm formation in isolated areas. Textured samples had a significantly lower percentage coverage after up to 21 days. Their findings suggest that the topographical features of the surface provide a physical obstacle to the expansion of clusters of bacterial cells and hence biofilm.

Hochbaum studied the ordering of Gram positive and negative bacterial cells, at the single cell level, induced by nanometre-scale periodic surface features [60]. They showed that as the surface periodicity created confined spaces of dimensions approaching those of the size of bacterial cells the bacterial interactions changed significantly. They also demonstrated the ability to control cell adhesion by adjusting the nanoscale topological features of a substrate material. Bacterial strains of $P$. aeruginosa (strain PA14), Bacillus subtilus (strain 3610) and Escherichia coli (strain W3110) were all tested and shown to assemble in similar ways. This study was extended further by Epsein where nanoscale polymer posts were fabricated in epoxy and polyurethanes and seeded with a culture of P.aeruginosa (strain PA14) before incubation for various time periods [61]. The study not only assessed the effect of surface texture but also the stiffness of topological features. It was found that bacteria attach in different configurations. The bacteria tended to be aligned with the posts normal to the substrate when their pitch was small $(900 \mathrm{~nm})$. The bacteria aligned in the orthogonal direction with increasing pitch posts, lying in the plane of the substrate. The studies demonstrated the ability to drive bacterial assembly using a two dimensional gradient substrate. It also confirmed that decreasing post pitch drives the bacterial cells to align normal to the substrate which is related to the wall to wall gap rather than the post diameter or post pitch. As post pitch further decreases attachment is random and disordered. No orientation is observed when the pitch size results in spacing longer than the length of a bacterial cell. They also studied the effect of the effective stiffness of the topological features and found that biofilms cultured on $2 \mathrm{GPa}$ and

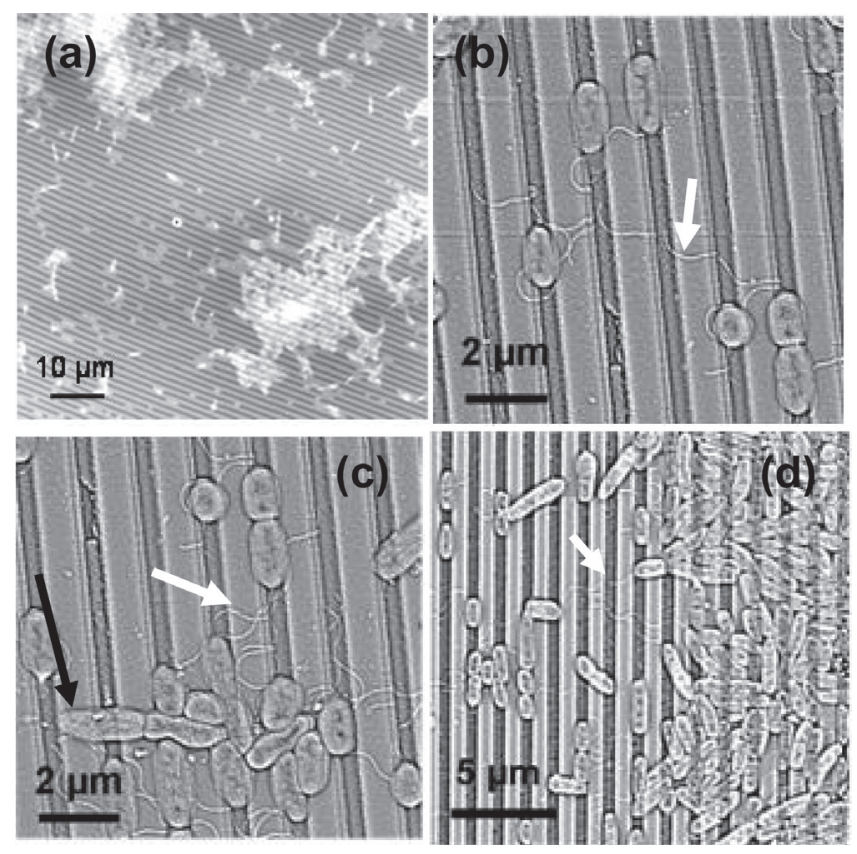

Figure 3: AFM images showing the retention of $P$. fluroscens retained on surfaces with different nano-topography. Reprinted with permission from [58] Copyright 2007 American Chemical Society.
$500 \mathrm{MPa}$ nanoarrays were larger than on corresponding flat surfaces, however there was a decrease in biofilm formation with $20 \mathrm{MPa}$ nanoarrays. It was concluded that this is most likely due an inhibitory effect caused by the low effective stiffness experienced by cells interacting with the nanoarrays. This study suggests a potential new strategy with nanoarrays mimicking extremely compliant flat surfaces offering promise for applications controlling biofilm accumulation. $\mathrm{Xu}$ also showed that an ordered nanoscale topography inhibits bacterial adhesion and subsequent biofilm formation by assessing the adhesion of staphylococci to submicron textured poly (urethane urea) films [62]. The patterns consisted of an ordered array of round pillars with a diameter of $400 / 500 \mathrm{~nm}$ and separation distance of $400 / 500 \mathrm{~nm}$. It was hypothesised that the submicron surface textures can reduce the adhesion of bacteria due to a decrease in surface area for contact with bacteria and this can subsequently inhibit biofilm formation. Bacterial adhesion was measured using a rotating disk system over a shear stress range of $0-13.2 \mathrm{dyn}^{-2}$ with textured samples and a control smooth sample before being stained and analysed using fluorescent microscopy. As the pillar separation distance is of sub-bacterial dimension the bacteria were restricted to only contacting the tops of the pillars. This reduced the interaction with the surface making them easier to be removed with a flow of fluid. A reduction in bacterial adhesion of up to $90 \%$ was demonstrated when compared to smooth surfaces. The patterned surfaces didn't show any signs of $S$. aureus biofilm formation until day 21 compared with day 7 with the smooth surfaces with a mean coverage of $7 \%$ compared with $54 \%$.

The large amount of research examining the influence of different nanoscale topographies, and patterning of surface chemistries and mechanical properties, on microbial adhesion and biofilm formation has demonstrated the tremendous potential for the nanoscale control enabled by fibre fabrication technologies such as electrospinning. The laying down of fibres on a surface or the formation of fibre mats are efficient methods to create multifunctional surfaces which permit control of microbial attachment and the impact of biofilms.

\section{Bacterial Interactions with Nanofibres}

The advent of nanotechnology and improved fabrication techniques triggered extensive research on the control of microbial adhesion by nanostructured surfaces. However, conspicuous by its absence, is a research focus on microbial adhesion and subsequent biofilm formation at nanofibre structures, given their industrial and clinical importance. Indeed there are very few quantitative studies examining the adhesion of micro-organisms to nanofibre textured surfaces. As at any surface there are a number of mechanisms that govern the adhesion of bacteria to nanofibres. These can be physio-chemical or specific and are influenced by the surface chemistry and the morphology of the fibres and the fibre mat. Physio-chemical interactions will have contribution from electrostatic and van der Waals forces and hydrophobicity as described by DLVO and extended DLVO theories. Specific interactions involve macromolecules at the bacterial surfaces that bind to specific sites at host and material surfaces. Surface appendages such as slime layers, fimbriae and pili can also contribute to bacterial surface interactions. For a more detailed discussion on bacterial adhesion to surfaces and the influence of material see Ploux [63] and Ubbink and ScharZammaretti [64]. The area of contact between the interacting cell and the surface is an important determinant of the size of the interaction forces; the greater the area of contact the larger number of interacting surface groups or molecular structures contributing to the bacterial cell attachment. When considering adhesion at planar surfaces the surface roughness and the deformation of the cell boundaries on contact with 
the surface will influence the area of contact between the cell and the surface. At surfaces covered in nanofibres the actual surface area of contact may be significantly reduced due to the void areas between the fibres. However, as previously stated the interaction of bacteria with fibrous structures requires more research. Kargar investigated the interaction of $P$. aeruginosa with nanofibre-textured surfaces [65]. They used highly aligned polystyrene nanofibres of different diameters and varying gap size fabricated using a fibre extrusion technique. They selected 3 fibre diameters, one smaller than the diameter of the organism, one equal to the diameter of the organism and one greater in diameter to that of the organism. Similarly, the gap size between fibres was selected with a gap sizer smaller than, equal to and greater than that of the organism. Samples were submerged in a suspension of $P$. aeruginosa and SEM was used to image the samples. The images were analysed, using the total number of bacteria per fibre length to quantify the total adhesion density. To further analyse the state of adhesion bacteria were characterised according to their alignment with the fibres and spacing. They found that the total adhesion density increases both with fibre diameter and spacing distance. This study used aligned fibres to easier understand the interaction with the fibres themselves. The absence of a control sample with a smooth surface limits the findings to how the bacteria interact with the nanofibre textured surface with no comparison to smooth surfaces.

Abriago investigated the effect of fibre diameter on bacterial attachment, proliferation and growth at electrospun fibre constructs (Figure 4) [66]. In their study varying concentrations of polystyrene (PS) in DMF were used to electrospin fibres of different diameter from $300 \mathrm{~nm}$ to $3000 \mathrm{~nm}$. Electrospun meshes were then tested against $E$. coli, $P$. aeruginosa and $S$. aureus both in solution and on agar plates. Their work demonstrated that the fibre diameter influences bacterial proliferation. An average fibre diameter close to that of the bacteria offered the best support for bacterial adhesion and proliferation. Rod shaped cells tended to wrap themselves around fibres with a smaller diameter than their length limiting the ability of the cells to bridge gaps between fibres and form colonies (Figure 5). Round cells tended to proliferate through nanofibrous substrates yet when the diameter was larger they were found to have adhered to the surface. Again, these findings were limited by the absence of a smooth control samples. In a further study Abriago investigated the bacterial response to different surface chemistries of electrospun nanofibres [67]. Polystyrene (PS) nanofibres were electrospun and plasma coated with a number of different monomers including allylamine (ppAAm), acrylic acid (ppAAc), 1,7-octadiene (ppOct) and 1,8-cineole (ppCo). The same techniques as the previous paper were used to characterise bacterial interactions with the fibres [66]. The plasma coating did not induce a significant change in fibre morphology. The surface chemistry was found to have a significant effect on bacterial adhesion and proliferation. A ppAAm coating (hydrophilic and rich in amine positively charged groups) resulted in the highest attraction of viable E. coli cells forming colonies and clusters across the interstices of the mesh. There was a significantly lower number of E. coli cells found on fibres with a hydrophilic, negatively charged ppAAc coating. The cells spread throughout the fibrous network. Fibres with a hydrophobic ppOct coating were found to have a higher proportion of live cells when compared to untreated PS fibres forming clusters at fibre crossovers. The ppCo coating had no inhibitory effect although a high proportion of dead isolated bacterial cells were found to have adhered to the fibres. Cells were wrapped around fibres with no clusters at fibre crossovers or across the interstices of the mesh. The results demonstrate the effect of surface chemistry on the interaction between bacteria and nanofibres
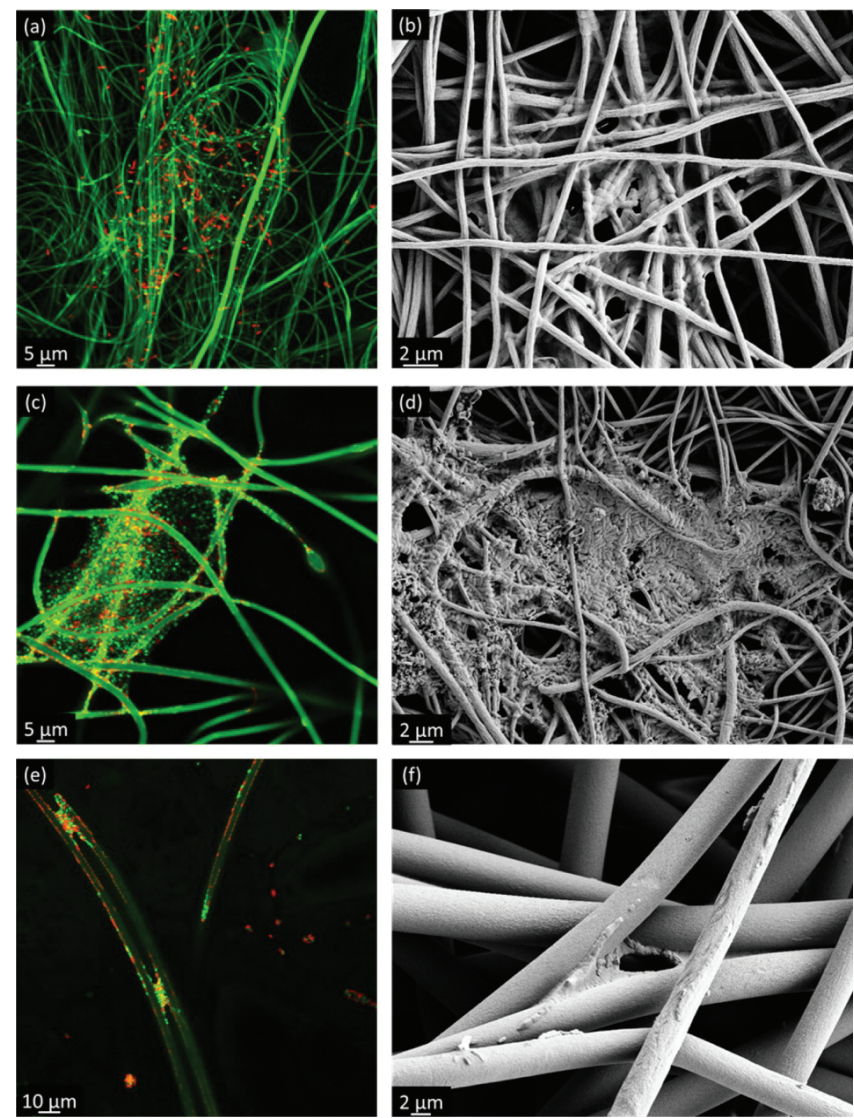

Figure 4: Confocal ( $a, b$ and c) and SEM (d, e and f) images of E. coli cells interacting with electrospun Polystyrene meshes with varying fibre diameters. (a,b) Average diameter $=500 \pm 200 \mathrm{~nm}$; (c, d) Average diameter $=1000 \pm 100$ $\mathrm{nm}$; (e, f) Average diameter $=3000 \pm 1000 \mathrm{~nm}$. Reprinted with permission from [66]. Copyright 2015 American Chemical Society.
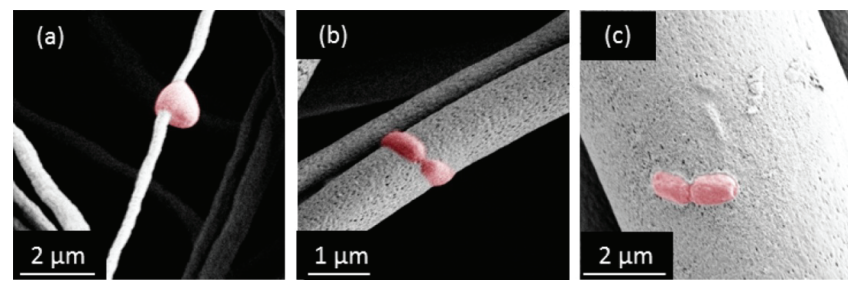

Figure 5: SEM images of E. coli cells (red) adhered to Polystyrene fibres of average diameter (a) $0.3 \mu \mathrm{m}$ (b) $1 \mu \mathrm{m}$ (c) $5 \mu \mathrm{m}$. Reprinted with permission from [66]. Copyright 2015 American Chemical Society.

offering a further parameter to be altered during electrospinning fibre fabrication to meet a specific anti-microbial attachment application.

\section{Conclusion}

In conclusion there has been extensive research studying the interaction between micro-organisms and nano and micro textured surfaces. However, there is very little research focussing on the interaction and adhesion of micro-organisms to micro- and nanofibres. An initial study on microbial colonisation of fibres used a fibre extrusion fabrication technique and therefore obtained extremely aligned fibres with defined diameter and gap distance, this is not always obtainable and usually undesirable in electrospun non-wovens. 
To move the technology forward in areas such as tissue engineering and wound healing the interaction between micro-organisms and materials fabricated using electrospinning have been briefly considered in studies by Abriago studying the effect of fibre diameter and the surface chemistry of polystyrene nanofibres. To further increase the understanding of bacterial interaction with nanofibres the studies need to be extended for different materials with different stiffness' and mechanical properties. Different inoculation techniques can be used such as a static, parallel plate flow chamber and rotating disk. By applying shear stress the understanding can be extended to biofilm formation. To visualise the interactions SEM and confocal microscopy can be used to assess orientation and coverage of adhering cells. To further assess the adhesion properties of the nanofibres single cell AFM can be used with force curve measurements to measure the adhesion force. These findings can be compared with the interactions of bacteria with planar surfaces and other nanostructured surfaces. This research will be able to guide the design of electrospun non-wovens and improve their microbial control in a broad range of applications. Tissue engineering scaffolds can be optimised to reduce microbial colonisation with the tantalising possibility of promoting mammalian cell adhesion while minimising the risk of infection by inhibiting bacterial cell adhesion through fine tuning of the nanofibrous material structure. Similar characteristics are also desirable in wound dressings. A more rigorous understanding of the influence of nanofibre structure on microbial adhesion will also help with the development of systems susceptible to biofilm formation such as filtration systems, where by reducing the adherent cells or controlling colony morphology and mechanical properties formation of biofilm can be inhibited or slowed.

\section{Acknowledgment}

This work is part-funded by the European Social Fund (ESF) through the European Union's Convergence programme administered by the Welsh Government, UK.

\section{References}

1. Sill TJ, von Recum HA (2008) Electrospinning: applications in drug delivery and tissue engineering. Biomaterials 29: 1989-2006.

2. Bhardwaj N, Kundu SC (2010) Electrospinning: a fascinating fiber fabrication technique. Biotechnol Adv 28: 325-347.

3. Jayasinghe SN (2011) Biojets in regenerative biology \& medicine. Mater Today 1: $202-211$.

4. Garg K, Bowlin GL (2011) Electrospinning jets and nanofibrous structures. Biomicrofluidics 5: 13403

5. Cui W, Zhou Y (2010) Electrospun nanofibrous materials for tissue engineering and drug delivery. Sci Technol Adv Mater 1: 014108.

6. Hassiba AJ, El Zowalaty, Nasrallah GK (2016) Review of recent research on biomedical applications of electrospun polymer nanofibers for improved wound healing. Nanomedicine 11: 715-737.

7. Chang C (2016) Development of Electrospun Lignin-based Fibrous Materials for Filtration Applications. BioResources 1: 2202-2213.

8. Strutt JW (1878) On the instability of jets. Proc London Math Soc 10: 4-13.

9. Cooley JF (1900) Improved methods of and apparatus for electrically separating the relatively volatile liquid component from the component of relatively fixed substances of composite fluids. Pat GB 11: 63-85.

10. Taylor G (1964) Disintegration of water drops in an electric field. Proc R Soc 280: 383-397.

11. Taylor G (1969) Electrically driven jets. Proc R Soc. 31: 453-475.

12. Yarin AL, Koombhongse $S$ (2001) Taylor cone and jetting from liquid droplets in electrospinning of nanofibers. J Appl Phys 90: 4836-4846.

13. Dosunmu OO, Chase GG, Kataphinan W, Reneker DH (2006) Electrospinning of polymer nanofibres from multiple jets on a porous tubular surface. Nanotechnology 17: 1123-1127.
14. Jirsak O, Sanetrnik F, Lukas D (2009) Method of nanofibres production from a polymer solution using electrostatic spinning and a device for carrying out the method. AIP 90: 4836

15. Wee-Eong T, Ryuji I (2011) Technological advances in electrospinning of nanofibers. Sci Technol Adv Mater 1: 13002

16. Lannutti J, Reneker D, Ma T (2007) Electrospinning for tissue engineering scaffolds 27: 504-509.

17. Nisbet DR, Forsythe JS, Shen W, Finkelstein DI, Horne MK (2009) Review paper: a review of the cellular response on electrospun nanofibers for tissue engineering. J Biomater Appl 24: 7-29.

18. Mi HY, Salick MR, Jing X (2015) Electrospinning of unidirectionally and orthogonally aligned thermoplastic polyurethane nanofibers: Fiber orientation and cell migration. J Biomed Mater Res 10: 593-603.

19. Li WJ, Laurencin CT, Caterson EJ, Tuan RS, Ko FK (2002) Electrospun nanofibrous structure: a novel scaffold for tissue engineering. J Biomed Mater Res 60: 613-621.

20. Gentile P, Chiono V (2014) An overview of poly(lactic-co-glycolic) Acid (PLGA) based biomaterials for bone tissue engineering. Int J Mol Sci 15: 3640-3659.

21. Petrigliano FA, Arom GA (2014) In Vivo Evaluation of Electrospun Polycaprolactone Graft for Anterior Cruciate Ligament Engineering. Tissue Eng 2: 150.

22. Kucinska-Lipka J, Gubanska I (2015) Fabrication of polyurethane and polyurethane based composite fibres by the electrospinning technique for soft tissue engineering of cardiovascular system. Mater Sci Eng 46: 166-176.

23. Naghavi S, Alhosseini F, Moztarzadeh S, Kargozar M (2015) Development of Polyvinyl Alcohol Fibrous Biodegradable Scaffolds for Nerve Tissue Engineering Applications:In In Vitroln Study. Int J Polym Mater Polym Biomater 6: 474-480.

24. Baek J, Sovani S, Glembotski NE (2016) Repair of Avascular Meniscus Tears with Electrospun Collagen Scaffolds Seeded with Human Cells. Tissue Eng 2: 284.

25. Anselme K, Davidson P, Popa AM, Giazzon M, Liley M, et al. (2010) The interaction of cells and bacteria with surfaces structured at the nanometre scale. Acta Biomater 6: 3824-3846.

26. Tamayol A, Akbari M, Annabi N, Paul A, Khademhosseini A, et al. (2013) Fiberbased tissue engineering: Progress, challenges, and opportunities. Biotechnol Adv 31: 669-687.

27. Li M, Mondrinos MJ, Gandhi MR, Ko FK, Weiss AS, et al. (2005) Electrospun protein fibers as matrices for tissue engineering. Biomaterials 26: 5999-6008.

28. Gao Y, Truong YB, Zhu Y (2014) Electrospun antibacterial nanofibers: Production, activity, and in vivo applications. J Appl Polym Sci 13: 9041-9053.

29. Zhang Y, Lim CT, Ramakrishna S, Huang ZM (2005) Recent development of polymer nanofibers for biomedical and biotechnological applications. J Mater Sci Mater Med 16: 933-946.

30. Jannesari M, Varshosaz J (2011) Composite poly(vinyl alcohol)/poly(viny acetate) electrospun nanofibrous mats as a novel wound dressing matrix fo controlled release of drugs. Int J Nanomedicine 6: 993-1003.

31. Heunis TD, Dicks LM (2010) Nanofibers offer alternative ways to the treatment of skin infections. J Biomed Biotechnol 2010

32. Naseri N, Mathew AP (2015) Porous electrospun nanocomposite mats based on chitosan??? cellulose nanocrystals for wound dressing: effect of surface characteristics of nanocrystals. Cellulose 2: 521-534

33. Lowe A, Bills J, Verma R, Lavery L, Davis K, et al. (2015) Electrospun nitric oxide releasing bandage with enhanced wound healing. Acta Biomater 13: 121 130.

34. Augustine R, Dominic EA, Reju I, Kaimal B, Kalarikkal N, et al. (2015) Electrospun poly(Îlu-caprolactone)-based skin substitutes: In vivo evaluation of wound healing and the mechanism of cell proliferation. J Biomed Mater Res B Appl Biomater 103: 1445-1454.

35. Bonvallet PP, Schultz MJ (2015) Microporous Dermal-Mimetic Electrospun Scaffolds Pre-Seeded with Fibroblasts Promote Tissue Regeneration in FullThickness Skin Wounds. PLoS One 10: e0122359.

36. Zahedi P, Rezaeian I, Ranaei-Siadat S (2010) A review on wound dressings with an emphasis on electrospun nanofibrous polymeric bandages. Polym Adv Technol 2: 77-95. 
Citation: Mortimer CJ, Burke L, Wright CJ (2016) Microbial Interactions with Nanostructures and their Importance for the Development of Electrospun Nanofibrous Materials used in Regenerative Medicine and Filtration. J Microb Biochem Technol 8: 195-201. doi: 10.4172/1948-5948.1000285

37. Harding KG, Morris HL (2002) Healing chronic wounds. thebmj 324: 160.

38. Rujitanaroj P, Pimpha N, Supaphol P (2008) Wound-dressing materials with antibacterial activity from electrospun gelatin fiber mats containing silver nanoparticles. Polymer 49: 4723-4732.

39. Lakshman LR, Shalumon KT (2010) Preparation of silver nanoparticles incorporated electrospun polyurethane nano-fibrous mat for wound dressing. J Macromol Sci 47: 1012-1018.

40. Khulbe K, Feng C, Ismail A (2015) Development of Large-Scale Industrial Applications of Novel Membrane Materials: Carbon Nanotubes, Aquaporins, Nanofibers, Graphene, and Metal-Organic Frameworks in Membrane Fabrication. CRC Press 11: 383-436.

41. Zhang S (2009) Design of ultra-fine nonwovens via electrospinning of Nylon 6 : Spinning parameters and filtration efficiency. Mater Des 30: 3659-3666.

42. Patanaik A (2010) Performance evaluation of electrospun nanofibrous membrane. J Memb Sci 35: 136-142.

43. Yun KM, Hogan CJ, Matsubayashi Y (2007) Nanoparticle filtration by electrospun polymer fibers. Chem Eng Sci 6: 4751-4759.

44. Nicosia A, Gieparda W, Foksowicz-Flaczyk J (2015) Air filtration and antimicrobial capabilities of electrospun PLA/PHB containing ionic liquid. Sep Purif Technol 15: 154-160.

45. Costerton JW, Stewart PS, Greenberg EP (1999) Bacterial biofilms: a common cause of persistent infections. Science 284: 1318-1322.

46. Donlan RM1 (2002) Biofilms: microbial life on surfaces. Emerg Infect Dis 8: 881-890.

47. Hall-Stoodley L, Costerton JW, Stoodley P (2004) Bacterial biofilms: from the natural environment to infectious diseases. Nat Rev Microbiol 2: 95-108.

48. Xu L, Siedlecki CA (2012) Acta Biomaterialia Submicron-textured biomaterial surface reduces staphylococcal bacterial adhesion and biofilm formation. Acta Biomater 8: 72-81.

49. Guélon T (2011) Advances in biofilm mechanics in Biofilm Highlights. Springer 12: 111-139.

50. Otto K (2008) Biophysical approaches to study the dynamic process of bacterial adhesion. Res Microbiol 159: 415-422.

51. O'Toole GA, Kolter R (1998) Initation of biofilm formation in Pseudomans fluorescens WC365 proceeds via multiple, convergent signalling pathways: a genetic analysis. Mol Microbiol 28: 449-461.

52. Otto K, Silhavy TJ (2002) Surface sensing and adhesion of Escherichia coli controlled by the Cpx-signaling pathway. Proc Natl Acad Sci U S A 99: $2287-$ 2292.
53. Camesano TA (2007) Measuring bacterial adhesion at environmental interfaces with single-cell and single-molecule techniques. Adv Water Resour 30: 14701491.

54. Hoa XD, Kirk AG (2007) Towards integrated and sensitive surface plasmon resonance biosensors: A review of recent progress. Biosens Bioelectron 2: 151-160.

55. Xu LC, Siedlecki CA (2012) Submicron-textured biomaterial surface reduces staphylococcal bacterial adhesion and biofilm formation. Acta Biomater 8: 72 81.

56. Whitehead KA, Colligon J (2005) Retention of microbial cells in substratum surface features of micrometer and sub-micrometer dimensions. Colloids Surfaces B Biointerfaces 4: 129-138.

57. Edwards KJ, Rutenberg AD (2001) Microbial response to surface microtopography?: the role of metabolism in localized mineral dissolution.

58. Díaz C, Schilardi PL, Salvarezza RC, de Mele MF (2007) Nano/microscale order affects the early stages of biofilm formation on metal surfaces. Langmuir 23: 11206-11210.

59. Chung KK (2007) Impact of engineered surface microtopography on biofilm formation of Staphylococcus aureus. Biointerphases 7: 89-94.

60. Hochbaum Al, Aizenberg J (2010) Bacteria pattern spontaneously on periodic nanostructure arrays. Nano Lett 10: 3717-3721.

61. Epstein AK, Hochbaum Al, Kim P, Aizenberg J (2011) Control of bacteria biofilm growth on surfaces by nanostructural mechanics and geometry. Nanotechnology 2: 494007

62. Xu LC, Siedlecki CA (2012) Submicron-textured biomaterial surface reduces staphylococcal bacterial adhesion and biofilm formation. Acta Biomater 8: 7281.

63. Ploux L, Ponche A, Anselme K (2010) Bacteria/material interfaces: role of the material and cell wall properties. J Adhes Sci Technol 2: 2165-2201.

64. Ubbink J, Schär-Zammaretti P (2007) Colloidal properties and specific interactions of bacterial surfaces. Curr Opin Colloid Interface Sci 1: 263-270.

65. Kargar M, Wang J, Nain AS (2012) Controlling bacterial adhesion to surfaces using topographical cues: a study of the interaction of Pseudomonas aeruginosa with nanofiber-textured surfaces. Soft Matter 8: 10254.

66. Abrigo M, Kingshott P, McArthur (2015) Electrospun polystyrene fiber diameter influencing bacterial attachment, proliferation, and growth. ACS Appl Mater Interfaces 7: 7644-7652

67. Abrigo M, Kingshott P, McArthur (2015) Bacterial response to different surface chemistries fabricated by plasma polymerization on electrospun nanofibers. Biointerphases 10: 04A301. 